\title{
Sleep Problems in Autism Spectrum Disorder: A Literature Review
}

\author{
Arlene Mannion • Geraldine Leader
}

Published online: 27 November 2013

(C) Springer Science+Business Media New York 2013

\begin{abstract}
The purpose of this literature review is to provide an overview of the research on sleep problems in autism spectrum disorder. Topics explored are the prevalence of sleep problems, the importance of studying sleep problems, as well as the objective and subjective measures used to measure sleep. Research on the relationships between sleep problems and parental stress and psychological distress, developmental regression, language and communication, adaptive behavior, autism severity, challenging behavior, comorbid psychopathology, attention deficit/hyperactivity disorder, gastrointestinal symptoms, epilepsy, and sensory issues is also discussed. Age-related variations in sleep problems are also examined. Finally, recommendations for treatment are given as well as areas where future research is needed.
\end{abstract}

Keywords Autism spectrum disorders · Sleep problems · Comorbidity · Treatment

\section{Introduction}

In their recent review of comorbidity and autism, Matson and Goldin (2013) discussed the development of a new field of study: comorbidity with autism spectrum disorder (ASD). The authors included a list of questions that need to be addressed including determining the most common comorbid disorders, how symptoms are expressed, how best to assess and diagnose these disorders, and what intervention strategies are likely to

This research was conducted by the first author under the supervision of the second author in partial fulfillment of the requirements for her $\mathrm{PhD}$ degree in ABA at NUI, Galway.

A. Mannion · G. Leader $(\bowtie)$

Irish Center for Autism and Neurodevelopmental Research, School of Psychology, National University of Ireland, Galway, Ireland

e-mail: geraldine.leader@nuigalway.ie be most efficacious. The aim of the current paper is to provide an overview of some of the possible answers to these questions for the topic of sleep problems in ASD. Matson and Goldin (2013) found sleep disturbance to be the second most common comorbid physical condition studied, after epilepsy. While research is increasing in the area of sleep problems in autism, there is a need for more research. This paper aims to provide an overview of the key issues in this area for researchers and clinicians alike.

\section{Sleep Problems and ASD Comorbidity}

\section{Prevalence}

High prevalence rates have been found for sleep problems in ASD in recent research studies. Rzepecka et al. (2011) reported sleep problems in $77.2 \%$ of children with ASD. Consistent with this finding, Mannion et al. (2013) found that $80.9 \%$ of children with ASD had sleep problems. Goldman et al. (2011) found a smaller prevalence of sleep problems, where $33 \%$ of their sample was identified as poor sleepers. Park et al. (2012) found that $47 \%$ of children with ASD had at least one sleep problem.

Importance of Studying Sleep Problems

Sleep affects not only the child, but the entire family unit. Sleep is essential for daily functioning. Relationships have been found between lack of sleep and daytime challenging behavior in individuals with ASD. Goldman et al. (2011) found that poor sleepers with ASD had more behavioral problems than good sleepers with autism. Understanding the relationship between sleep and its correlates will mean having a better understanding of how best to provide treatment for sleep problems in those with ASD. 


\section{Assessment}

\section{Objective Measures Used to Assess Sleep}

Hodge et al. (2012) conducted a literature on the objective and subjective in children with ASD. The review included the following objective measures: (1) polysomnography, (2) actigraphy, and (3) videosomnography. Polysomnography provides information on sleep stages and the physiologic activities that occur during sleep (e.g., eye movements, muscle activity, brainwave activity, oxygen saturation levels) (Hodge et al., 2012). Malow et al. (2006) combined polysomnography with parental report data to investigate sleep problems.

Actigraphy is an objective measure of sleep that is conducted by monitoring night time movement of the limbs (Hodge et al. 2012). It involves wearing a wristwatch-like microcomputer that records motion. Meltzer et al. (2012) have provided a thorough review on the use of actigraphy for assessment in pediatric sleep research. This may be useful for researchers embarking on actigraphy research. Wiggs and Stores (2004) combined parental report with actigraphy. Adkins et al. (2012) combined actigraphy with the Children's Sleep Habits Questionnaire (CSHQ) (Owens et al. 2000) in order to investigate the effectiveness of a standardized pamphlet on insomnia. Similarly, GoodlinJones et al. (2008b) combined actigraphy with the CSHQ and sleep diaries. Goodlin-Jones et al. (2008a) found that actigraphy, the CSHQ, and parent sleep logs were significantly correlated. Meltzer (2011) assessed sleep using actigraphy on mothers, fathers, and children in order to investigate the relationship between sleep and depressive symptoms in parents of children with ASD.

Souders et al. (2009) commented that children with autism often have sensory challenges, such as tactile defensiveness. Souders et al. (2009) piloted an alternative approach to address these concerns by placing the actigraph in a pocket in the upper sleeve arm area of a pajama top. This method of data collection was found to be as reliable as actigraphs worn on the wrist, and was found to be tolerable for children with autism. This alternative validated method of actigraph placement was also implemented by Adkins et al. (2012).

Videosomnography is an objective measure of sleep in which a portable time-lapse video recording system is placed in the bedroom (Anders and Sostek 1976; Hodge et al. 2012). Sitnick et al. (2008) compared videosomnography with actigraphy. Findings were $94 \%$ overall agreement between the two measures. Actigraphy was shown to have poor agreement for detecting nocturnal awakenings, compared with video observations in pre-school aged children. The authors suggested exercising caution in using actigraphy as the only method of assessment. Hodge et al. (2012) commented that polysomnography and actigraphy may be difficult for some children with ASD to tolerate. The authors recommended that researchers consider using videosomnography as an alternative to or in combination with actigraphy.

\section{Subjective Measures to Assess Sleep}

Spruyt and Gozal (2011a) provided a comprehensive review of the currently available pediatric sleep questionnaires. While it is important that researchers investigating sleep problems in ASD use tools that have been piloted and validated for an ASD population, Spruyt and Gozal's review is a very useful starting point for researchers and clinicians. Spruyt and Gozal (2011b) also wrote a review on Dos and Don'ts of developing pediatric sleep questionnaires. Should a researcher not find a questionnaire suitable for their research, this review provides considerations that may be adhered to when designing a sleep assessment.

Three of the questionnaires that have been used with children with ASD include the Behavioral Evaluation of Disorders of Sleep (BEDS) (Schreck, 1998; Schreck et al. 2003), Children's Sleep Habits Questionnaire (CSHQ) (Owens et al., 2000), and the Family Inventory of Sleep Habits (FISH) (Malow et al., 2009). The BEDS was developed for use for children aged 5 to 12 years and consists of 107 items. It was recently used in Taylor et al. (2012). The CSHQ is a 33-item questionnaire for children aged 4 to 12 years. It has also been used for younger children from the age of 2 onwards (Goodlin-Jones et al. 2008a). It has recently been administered in Hollway et al. (2013) and Mannion et al. (2013). The FISH provides a quantitative scale of sleep habits, including bedtime routine, sleep environment, and parental interactions. It has been validated as a 12 -item scale. It was recently used in Malow et al. (2013).

In their review, Hodge et al. (2012) discussed the following subjective sleep measures: (1) sleep diaries, (2) single-item responses, and (3) CSHQ. The authors commented that there is modest evidence to suggest that a parent-report measure such as the CSHQ can be superior to single-item responses for assessing children's sleep quality. Hodge et al. (2012) encouraged researchers to use multiple and objective measures of children's sleep.

\section{Relationships Between Sleep Problems in ASD and Other Variables}

Hollway and Aman (2011a) conducted a comprehensive literature review on the sleep correlates of pervasive developmental disorders. The current paper aims to provide an overview of some of the key correlates in autism spectrum disorder specifically. To expand on Hollway and Aman (2011a), we will discuss the relationships between sleep problems in ASD and other variables that were not discussed in depth in Hollway and Aman (2011a), such as parental stress and 
psychological distress, gastrointestinal symptoms, and sensory issues. Where possible, new additions to the literature since 2011 will be discussed.

\section{Parental Stress and Psychological Distress}

Richdale et al. (2000) investigated sleep problems and family stress in individuals with intellectual disability. Richdale et al. (2000) found that the presence of sleep problems was associated with both the intensity and frequency of family hassles in their parents. Gallagher et al. (2009) investigated sleep quality in parents of children with developmental disabilities. The majority of parents were carers of children with autism ( $55 \%$ of all participants), while the remainder of parents cared for children with Down syndrome and other syndromes. The authors found that that parenting stress emerged as the strongest predictor of poor sleep quality in parents caring for children with developmental disabilities.

In the ASD literature, Hodge et al. (2013) examined the relationship between children's sleep and mother's mental health for mothers of children with and without ASD. The authors found that children's sleep significantly predicted maternal mental health, maternal sleep, and maternal stress. The authors found that mothers of children with ASD reported more problems related to their children's sleep, their own sleep, greater stress, and poorer mental health.

Meltzer (2011) recruited both mothers and fathers in their study and used actigraphy to investigate factors associated with parent depressive symptoms. Maternal depressive symptoms were associated with shorter child sleep quantity, increased child sleep disturbances, shorter maternal sleep quantity, and poorer maternal sleep quality. Child sleep quantity was found to predict maternal depressive symptoms. For fathers, paternal depressive symptoms were associated with increased child sleep disturbances and poorer paternal sleep quality. Sleep quality predicted paternal depressive symptoms.

\section{Developmental Regression}

Cortesi et al. (2010) commented on the possible relationship between sleep problems and developmental regression. Hollway et al. (2013) found that there were more sleep problems in children who had regressed than those who had no developmental regression. Hansen et al. (2008) reported that no significant differences were found between the children with or without regression in terms of sleep problems. This is an area where more research is needed.

\section{Language and Communication}

When examining the relationship between sleep problems and communication, it was reported that communication problems were predicted by increased sensitivity to environmental stimuli (such as noise, light etc.) in the bedroom and screaming at night (Schreck et al. 2004). This finding is consistent with Taylor et al. (2012) who reported that more hours slept per night combined and fewer hours of night waking with screaming and more sensitivity to sleeping environment disturbances predicted children's higher communication scores. The authors explained those who slept more hours without waking each night showed better communication skills. Hollway et al. (2013) found that the communication domain of the Vineland Adaptive Behavior Scales (VABS) (Sparrow et al. 1984) predicted sleep dysfunction. An inverse association was found between communication and sleep duration. Gabriels et al. (2005) found a significant correlation between nonverbal IQ scores and sleep problems. Children with ASD and lower nonverbal IQs had more sleep problems than those with higher nonverbal IQs. Sikora et al. (2012) found no significant difference between those with mild and moderate sleep problems to those with severe sleep problems in communication scores.

\section{Adaptive Behavior}

Hollway and Aman (2011a) found that neither daily living skills scores nor social behavioral skills scores on the VABS (Sparrow et al., 1984) predicted sleep problems. Taylor et al. (2012) investigated the relationship between sleep disruption and adaptive behavior. More hours slept per night and hours napped during the day predicted better adaptive behavior composite, motor skills scores, and socialization scores on the VABS. More hours slept per night predicted daily living skills. Sikora et al. (2012) found that those with moderate to severe sleep problems had the lowest VABS composite score when compared to those with mild sleep problems or good sleepers. For the Daily Living Skills domain, there was no difference between the good sleepers and those with mild sleep problems for both pre-school and school-aged children. However, there were differences between the good sleepers and those with moderate to severe sleep problems for preschool children. For the Socialization domain, there were differences in scores between the good sleepers and the sleep problems groups for school-aged children, but no differences were evidenced for the pre-school aged children. Schreck et al. (2004) reported that fewer hours of sleep per night predicted social skills deficits.

\section{Autism Severity}

In their literature review, Hollway and Aman (2011a) found that out of the 11 studies that they analyzed investigating the relationship between autism severity and sleep problems, autism symptom severity was significantly associated with sleep disturbance in $77 \%$ of the studies. The authors commented that children with more severe cases of autism 
spectrum disorder were more likely to develop sleep disturbances. This is supported by Mayes and Calhoun (2009) who reported that sleep problems increased with severity of autism symptoms and with severity of parent-reported symptoms. The authors also found that autism severity was the single most powerful predictor of sleep problems and explained $20 \%$ of the variance (Mayes and Calhoun, 2009). Schreck et al. (2004) reported that fewer hours of sleep per night predicted overall autism severity scores. Therefore, the fewer hours slept, the more severe were the symptoms of autism. Hollway et al. (2013) found that the core symptoms of autism and autism subtype predicted sleep quality in children with ASD.

\section{Challenging Behavior}

There is a large amount of research in support of the relationship between sleep problems and challenging behavior. Cortesi et al. (2010) commented on the importance of intervening with children with ASD and sleep problems in order to improve daytime functioning. Gregory and Sadeh (2012) discussed behavioral and sleep problems in typically developing children and adolescents in their review and commented that behavioral problems such as aggression, conduct disorders, and addiction have received less attention in the literature. We must consider the role that intellectual disability plays in children with ASD who display sleep problems. In a sample of children with intellectual disability, children with intellectual disability and severe sleep problems showed more severe levels of daytime problem behavior, such as aggression, noncompliance, and hyperactivity than those without sleep problems (Didden et al. 2002). Sleep problems were associated with the presence of problem behavior in children with and intellectual disability (Richdale et al. 2000).

In the ASD literature, Goldman et al. (2011) found that poor sleepers had a higher percentage of behavioral problems (such as stereotypy and self-injurious behavior) than good sleepers. Schreck et al. (2004) reported that stereotypic behavior was predicted by fewer hours of sleep per night and screaming during the night. Sleep problems increased with severity of parent-reported symptoms, such as oppositional behavior, aggression, and explosiveness (Mayes and Calhoun, 2009). Medication usage, sleep problems, and anxiety accounted for $42 \%$ of the variance in challenging behavior, with sleep problems being the strongest predictor (Rzepecka et al. 2011).

Gabriels et al. (2005) reported a significant correlation between sleep problem total score and repetitive behaviors. This is supported by Goldman et al. (2009), who also reported a relationship between sleep problems and repetitive behavior. Poor sleepers with ASD showed higher levels of compulsive and ritualistic behaviors than good sleepers with ASD. Nighttime fragmentation of sleep was significantly associated with total repetitive behavior as well as the individual scales of compulsive behaviors, need for sameness, and restricted behaviors.

Park et al. (2012) found that children with ASD and sleep problems were more likely to have aggressive behaviors and externalizing problems compared to children with ASD without sleep problems. In support, Sikora et al. (2012) found that children with ASD and sleep problems have more externalizing and internalizing behavior problems than those with ASD and no sleep problems. It was reported that children with moderate to severe sleep problems had greater behavior difficulties than those with mild to moderate sleep problems. In Hollway et al. (2013), the authors commented that they have data in preparation examining the relationship between sleep problems and aggression.

\section{Comorbid Psychopathology}

In their review, Gregory and Sadeh (2012) commented on the links between sleep and psychopathology is likely bidirectional. While their review was in relation to typically developing individuals and individuals with various psychological disorders, we must consider the possibility that this bidirectionality between sleep problems and psychopathology is a feature in individuals with ASD also. In the typically developing literature, Leahy and Gradisar (2012) discussed the bidirectional relationship between pediatric sleep and anxiety. Their review provided preliminary support that sleep problems may lead to the later development of anxiety in children and adolescents. The neurotypical literature also examined the possibility of a link between mild sleep disordered breathing and psychiatric disorders (Gold, 2011). In their commentary, Vgontas and Fernandez-Mendoza (2011) reported that Gold's review of the literature does not support the link due to methodological limitations in the studies included in Gold's article.

In the ASD literature, a number of studies have found links between sleep problems and comorbid psychopathology. Goldman et al. (2011) found that younger children who were poor sleepers displayed more hyperactivity and poorer eating habits than good sleepers. Poor sleepers had a higher percentage of problems overall. Mayes and Calhoun (2009) investigated variables related to sleep problems in children with ASD. Sleep disturbances was related to oppositional behavior, aggression, explosiveness, attention deficit, hyperactivity, anxiety, depression, and mood variability.

Hollway and Aman (2011a) conducted a review of sleep correlates in pervasive developmental disorders. With regards to internalizing behavior, the authors noted that the measures used in a number of studies that they reviewed used measures such as the Strengths and Difficulties Questionnaire (Goodman 2001) and the Youth Self Report (Achenbach 1991a), and Child Behavior Checklist (Achenbach 1991b) have been validated for typically developing children. 
Therefore, it is important that comorbid psychopathology measures are used that are validated for individuals with autism spectrum disorder, such as the Autism Spectrum Comorbidity-Children (ASD-CC) (Matson and González 2007). Mannion et al. (2013) investigated the relationship between sleep problems and comorbid psychopathology. The authors found that the under-eating and avoidant behavior subscales on the ASD-CC (Matson and González 2007) predicted the total score on the CSHQ.

Hollway and Aman (2011a) commented on the need for future research to examine the relationship between sleep, anxiety, and depression and on the importance of separating individuals with anxiety from individuals with depression. Their review found that internalizing behavior along with autism severity were the two strongest predictors of sleep disturbances with ASD. Therefore, this shows the importance of future research examining internalizing behavior as part of comorbid psychopathology.

Hollway et al. (2013) found that anxiety was associated with sleep disturbances in data analyzed from the Autism Treatment Network. Malow et al. (2006) found that anxious/ depressed behavior and affective problems correlated with sleep latency. Good sleepers with ASD showed fewer affective problems than poor sleepers with ASD. Park et al. (2012) examined the relationship between sleep problems and comorbid psychopathology in children with ASD and their unaffected siblings. Children with ASD who also had comorbid sleep problems were more likely to have withdrawal problems, somatizing problems, internalizing problems, and overall total behavior problems than children with ASD who did not have sleep problems. This is supported by Sikora et al. (2012) who found that children with ASD and sleep problems had more internalizing and externalizing behavior problems than those with ASD and no sleep problems.

\section{Attention Deficit/Hyperactivity Disorder}

In their review of sleep problems, Gregory and Sadeh (2012) discussed the research conducted investigating the relationship between sleep problems and attention deficit/hyperactivity disorder (AD/HD). In their review, Hollway and Aman (2011a) reported that externalizing behavior and sleep problems were associated in $70 \%$ of the comparisons of studies that the authors conducted. The authors commented on need for research to determine whether sleep deprivation influences externalizing behavior, whether externalizing behavior influences sleep, or whether there is a bidirectional relationship between the two.

Tsai et al. (2012) compared children with ASD to those with $\mathrm{AD} / \mathrm{HD}$, epilepsy, and to typically developing controls. Though the authors did not investigate the comorbidity between diagnoses, they did find that there were more current and lifetime sleep problems in children with ASD and AD/HD than in children with epilepsy. This was especially so for sleep problems like snoring and restless legs syndrome. Matson et al. (2013) conducted a thorough review of the relationship between $\mathrm{ASD}$ and $\mathrm{AD} / \mathrm{HD}$. In their discussion of possible sleep problems in those with ASD and a comorbid AD/HD diagnosis, the authors commented on the necessity for evaluation of sleep disorders. The authors commented that sleep problems should be treated prior to the re-evaluation of $\mathrm{AD} /$ HD symptoms to ensure that symptoms were not due to a primary sleep disorder.

\section{Gastrointestinal Symptoms}

In their recent review, Mannion and Leader (in press) commented on the comorbidity between sleep problems and gastrointestinal symptoms. In their previous research, Mannion et al. (2013) found that total number of gastrointestinal symptoms predicted sleep problems. Mannion and Leader (2013) found that $67.8 \%$ of children and adolescents with ASD had both sleep problems and gastrointestinal symptoms. The authors found that sleep problems also predicted gastrointestinal symptoms. Sleep disordered breathing and daytime sleepiness predicted both abdominal pain and bloating.

This finding of gastrointestinal symptoms predicting sleep problems is supported by Hollway et al. (2013). Hollway et al. (2013) found that gastrointestinal problems including constipation and diarrhea predicted total scores on the CSHQ, which was the same measure used by Mannion et al. (2013). Gastrointestinal symptoms predicted sleep anxiety in Hollway et al.'s (2013) research. This is supported by Mannion et al.'s (2013) finding of the relationship between gastrointestinal symptoms and sleep anxiety. The relationship between sleep problems and gastrointestinal symptoms is supported by Williams et al. (2012). The authors reported that abdominal pain predicted sleep anxiety. The relationship between sleep problems and gastrointestinal symptoms is one that needs to be further explored in future research.

\section{Epilepsy}

Hollway et al. (2013) found that epilepsy did not predict sleep disturbance in their study of children with ASD. Hollway et al. (2013) commented that the result is inconclusive in determining if epilepsy is part of a bidirectional theoretical framework on sleep dysfunction. Epilepsy did not predict sleep problems in Mannion et al. (2013). Tsai et al. (2012) found that sleep problems were more common in children with ASD and AD/ HD than with epilepsy. However, the authors commented on the comorbidity between ASD and AD/HD and epilepsy, and the importance of being aware of the occurrence of sleep problems in neurodevelopmental conditions.

In their review, van Golde et al. (2011) examined sleep disturbances in people with epilepsy. The authors commented 
on the complexity of the interaction between epilepsy and sleep problems. The article discussed the effect sleep disturbances can have on quality of life in individuals with epilepsy. Quality of life in individuals with ASD and comorbid epilepsy and sleep problems is an area of future research.

\section{Sensory Issues}

Goldman et al. (2011) found that sensory issues were more common in poor sleepers than in good sleepers. Hollway et al. (2013) found that children with ASD who were underresponsive, sensory seeking, and who displayed auditory filtering problems had more difficulty sleeping. Those who had lower scores or more impairments in taste and smell sensitivities were associated with increased sleep anxiety. The authors commented on the need for more research on physiological arousal, sensory sensitivities, and sensory seeking behavior in relation to sleep disturbance.

\section{Age-Related Variations in Sleep Problems}

Goldman et al. (2012) investigated the variations in sleep problems from childhood to adolescence. The authors reported that for all of the sleep problems, differences were found between children younger than 7 and children 11 years and older. The authors reported that sleep problems persist throughout the age span from early childhood through adolescence. Sleep problems also vary depending on the age of the child. Parents of younger children were more likely to report problems with sleep anxiety, bedtime resistance, night wakings, and parasomnias. The parents of adolescents reported more problems with falling asleep, getting enough sleep, and daytime sleepiness. Hollway et al. (2013) found an inverse association between age and sleep outcomes. The authors commented on the need for continued study in this area.

\section{Recommendations for Treatment}

Malow et al. (2012) provided a practice pathway for the identification, evaluation, and management of insomnia in children and adolescents with ASD. The consensus of sleep experts concluded as follows: (1) all children who have ASD should be screened for insomnia; (2) screening should be done for potential contributing factors, including other medical problems; (3) the need for therapeutic intervention should be determined; (4) therapeutic interventions should begin with parent education in the use of behavioral approaches as a first-line approach; (5) pharmacological therapy may be indicated in certain situations; and (6) there should be a follow-up after any intervention to evaluate effectiveness and tolerance of the therapy.
Adkins et al. (2012) investigated where a pamphlet alone could be used by parents to help their child's insomnia. It was determined that the pamphlet alone without further instruction for its use did not significantly improve children's sleep patterns. Sleep efficiency did show significant improvement but the authors commented that it was unlikely to be clinically significant. Malow et al. (2013) expanded on this study and provided sleep education for parents of children with ASD. Parent-based sleep education was found to be associated with improved sleep onset delay in children with ASD. Weiskop et al. (2005) also used parent training. The authors found that settling problems, night waking, and co-sleeping were effectively reduced. Parent-based sleep education is a very interesting area for future research.

When considering treatment fidelity, it is important to be aware of parental perceptions of efficacy. Williams et al. (2006) investigated parental perceptions of efficacy of sleep interventions. Behavioral strategies considered most effective by parents included establishing a bedtime routine, bringing the child into the parent's bed, wrapping the child, using a noise masker, and allowing the child to have toys in bed. Medication was used much less frequently than behavioral interventions.

Behavioral interventions have been used in many studies to reduce sleep problems in children with ASD. Moon et al. (2011) used the following behavioral strategies: faded bedtime with response cost and positive reinforcement for children with insomnia. Parents were provided with a treatment handbook and weekly telephone contact with a therapist. Mean sleep onset latency was reduced for all three children, and the results were generally maintained at 12 -week follow-up. Vriend et al. (2011) conducted a systematic review on the effectiveness of behavioral treatment of sleep problems in children with ASD. Standard extinction and scheduled awakenings met criteria for possibly efficacious interventions for sleep problems. More research is needed to determine the efficacy of graduated extinction, faded bedtime, stimulus fading, and chronotherapy. Turner and Johnson (2012) reviewed behavioral interventions for children with ASD and sleep problems under the age of 7 years old. The review evaluated antecedent-based procedures; such as bedtime fading, stimulus fading, and scheduled awakenings and consequence-based procedures; such as standard and graduated extinction. The authors reported that both antecedent and consequence-based interventions were in the emerging evidence strength category.

Where pharmacological treatment is necessary, Hollway and Aman (2011b) have provided a comprehensive literature review of pharmacological treatment of sleep disturbance in developmental disabilities. The authors concluded that melatonin appears to be the most widely assessed agent and safest choice for children with developmental disabilities. Wright et al. (2011) conducted a randomized controlled trial on the effectiveness of oral melatonin for sleep problems in children 
with ASD. In their letter to the editor, Guénolé and Baleyte (2011) commented on the need for assessing variables such as quality of life, parents' night time distress, and daily impairment and family stress.

\section{Future Research}

In their review of sleep problems with adults with intellectual disability, van de Wouw et al. (2012) reported that there is conflicting evidence concerning the association of ASD and sleep problems in adults with intellectual disability. The authors commented on the need for studies such objective data such as actigraphy. They also commented on the need for longitudinal research in adults. Therefore, it is clear that there is a need for research in adults with ASD to determine the prevalence of sleep problems, as well as the factors associated with sleep problems in this population.

Hollway et al. (2013) commented on the need for studies to focus on anxiety as anxiety was found to be the strongest predictor of sleep disturbance in their research. The authors also commented on the need for longitudinal studies to evaluate causal factors that may affect sleep problems. The need for longitudinal research on sleep is supported by Gregory and Sadeh (2012) who included measuring sleep in large-scale longitudinal studies as part of the research agenda the authors suggested. The authors also commented on the need to (1) use multi-methods (i.e., objective and subjective measures) to assess sleep, (2) conduct controlled experiments to establish the effects of sleep variations on emotional and behavioral difficulties, and (3) take an interdisciplinary approach to further understand the links between sleep and associated difficulties. Gregory and Sadeh (2012) suggested twin studies and collecting deoxyribonucleic acid of those participating in studies as two possible ways of conducting interdisciplinary research. They also commented on the need for additional sleep interventions studies.

Further research is also needed to explore the correlates between sleep problems and variables such as developmental regression and communication. Research is needed to investigate the relationship between sleep problems and comorbid conditions in ASD such as gastrointestinal symptoms and epilepsy.

\section{References}

Achenbach, T. M. (1991a). Manual for the Child Behavior Checklist 4-18 and 1991 profile. Burlington, VT: University of Vermont, Research Center for Children, Youth, \& Families.

Achenbach, T. M. (1991b). Manual for the Youth Self-Report and 1991 profile. Burlington, VT: University of Vermont, Research Center for Children, Youth, \& Families.
Adkins, K. W., Molloy, C., Weiss, S. K., Reynolds, A., Goldman, S. E., Burnette, C., et al. (2012). Effects of a standardized pamphlet on insomnia in children with autism spectrum disorders. Pediatrics, 130(Supple. 2), S139-S144. doi:10.1542/peds.2012-0900K.

Anders, T. F., \& Sostek, A. M. (1976). The use of time lapse video recording of sleep-wake behavior in human infants. Psychophysiology, 13(2), 155-158. doi:10.1111/j.1469-8986. 1976.tb00092.x.

Cortesi, F., Giannotti, F., Ivanenko, A., \& Johnson, K. (2010). Sleep in children with autistic spectrum disorder. Sleep Medicine, 11, 659-664. doi:10.1016/j.sleep.2010.01.010.

Didden, R., Korzilius, H., van Aperlo, B., van Overloop, C., \& de Vries, M. (2002). Sleep problems and daytime problem behaviors in children with intellectual disability. Journal of Intellectual Disability Research, 46(7), 537-547. doi:10.1046/j.1365-2788.2002.00404.x.

Gabriels, R. L., Cuccaro, M. L., Hill, D. E., Ivers, B. J., \& Goldson, E. (2005). Repetitive behaviors in autism: relationships with associated clinical features. Research in Developmental Disabilities, 26, 169-181. doi:10.1016/j.ridd.2004.05.003.

Gallagher, S., Phillips, A. C., \& Carroll, D. (2009). Parental stress is associated with poor sleep quality in parents caring for children with developmental disabilities. Journal of Pediatric Psychology, 35(7), 728-737. doi:10.1093/jpepsy/jsp093.

Gold, A. (2011). Functional somatic syndromes, anxiety disorders, and the upper airway: a matter of paradigms. Sleep Medicine Review, 15(6), 389-401. doi:10.1016/j.smrv.2010.11.004.

Goldman, S. E., McGrew, S., Johnson, K. P., Richdale, A. L., Clemons, T., \& Malow, B. A. (2011). Sleep is associated with problem behaviors in children and adolescents with Autism Spectrum Disorders. Research in Autism Spectrum Disorders, 5, 1223-1229. doi:10.1016/j.rasd.2011.01.010.

Goldman, S. E., Richdale, A. L., Clemons, T., \& Malow, B. A. (2012). Parental sleep concerns in autism spectrum disorders: variations from childhood to adolescence. Journal of Autism and Developmental Disorders, 42, 531-538. doi:10.1007/s10803-0111270-5.

Goldman, S. E., Surdyka, K., Cuevas, R., Adkins, K., Wang, L., \& Malow, B. A. (2009). Defining the sleep phenotype in children with autism. Developmental Neuropsychology, 34(5), 560-573. doi:10. 1080/87565640903133509.

Goodlin-Jones, B. L., Sitnick, S. L., Tang, K., Liu, J., \& Anders, T. F. (2008a). The Children's Sleep Habits Questionnaire in toddlers and pre-school children. Journal of Developmental and Behavioral Pediatrics, 29, 82-88. doi:10.1097/DBP.0b013e318163c39a.

Goodlin-Jones, B. L., Tang, K., Liu, J., \& Anders, T. (2008b). Sleep patterns in preschool-age children with autism, developmental delay, and typical development. Journal of the American Academy of Child and Adolescent Psychiatry, 47(8), 930-938. doi:10.1097/ CHI.0b013e3181799f7c.

Goodman, R. (2001). Psychometric properties of the Strengths and Difficulties Questionnaire. Journal of the American Academy of Child \& Adolescent Psychiatry, 40, 1337-1345. doi:10.1097/ 00004583-200111000-00015.

Gregory, A. M., \& Sadeh, A. (2012). Sleep, emotional and behavioral difficulties in children and adolescents. Sleep Medicine Reviews, 16, 129-136. doi:10.1016/j.smrv.2011.03.007.

Guénolé, F., \& Baleyte, J. (2011). Effectiveness of melatonin for sleep problems in autism spectrum disorders: evidence grows but research is still needed. Journal of Autism and Developmental Disorders, 41, 974-975. doi:10.1007/s10803-010-1111-y.

Hansen, R. L., Ozonoff, S., Krakowiak, P., Angkustsiri, K., Jones, C., Deprey, L. J., et al. (2008). Regression in autism: prevalence and associated factors in the CHARGE study. Ambulatory Pediatrics, 8(1), 25-31. doi:10.1016/j.ambp.2007.08.006.

Hodge, D., Hoffman, C. D., Sweeney, D. P., \& Riggs, M. L. (2013). Relationship between children's sleep and mental health in mothers 
of children with and without autism. Journal of Autism and Developmental Disorders, 43, 956-963. doi:10.1007/s10803-0121639-0.

Hodge, D., Parnell, A. M. N., Hoffman, C. D., \& Sweeney, D. P. (2012). Methods for assessing sleep in children with autism spectrum disorders: a review. Research in Autism Spectrum Disorders, 6, 1337-1344. doi:10.1016/j.rasd.2012.05.009.

Hollway, J. A., \& Aman, M. G. (2011a). Sleep correlates of pervasive developmental disorders: a review of the literature. Research in Developmental Disabilities, 32, 1399-1421. doi:10.1016/j.ridd. 2011.04.001

Hollway, J. A., \& Aman, M. G. (2011b). Pharmacological treatment of sleep disturbance in developmental disabilities: a review of the literature. Research in Developmental Disabilities, 32, 939-962. doi:10.1016/j.ridd.2010.12.035.

Hollway, J. A., Aman, M. G., \& Butter, E. (2013). Correlates and risk markers for sleep disturbance in participants of the autism treatment network. Journal of Autism and Developmental Disorders. doi:10. 1007/s10803-013-1830-y.

Leahy, E., \& Gradisar, M. (2012). Dismantling the bidirectional relationship between paediatric sleep and anxiety. Clinical Psychologist, 16 , 44-56. doi:10.1111/j.1742-9552.2012.00039.x.

Malow, B. A., Adkins, K. W., Reynolds, A., Weiss, S. K., Loh, A., Fawkes, D., et al. (2013). Parent-based sleep education for children with autism spectrum disorders. Journal of Autism and Developmental Disorders . doi:10.1007/s10803-013-1866-z.

Malow, B. A., Byars, K., Johnson, K., Weiss, S., Bernal, P., Goldman, S. E., et al. (2012). A practice pathway for the identification, evaluation, and management of insomnia in children and adolescents with autism spectrum disorders. Pediatrics, 130 (Suppl. 2), S106-S124. doi:10.1542/peds.2012-0900I.

Malow, B. A., Crowe, C., Henderson, L., McGrew, S. G., Wang, L., Song, Y., et al. (2009). A sleep habits questionnaire for children with autism spectrum disorders. Journal of Child Neurology, 24(1), 19-24. doi:10.1177/0883073808321044.

Malow, B. A., Marzec, M. L., McGrew, S. G., Wang, L., Henderson, L. M., \& Stone, W. L. (2006). Characterizing sleep in children with autism spectrum disorders: a multidimensional approach. Sleep, 29(12), 1563-1571.

Mannion, A., Leader, G., \& Healy, O. (2013). An investigation of comorbid psychological disorders, sleep problems, gastrointestinal symptoms, and epilepsy in children and adolescents with autism spectrum disorder. Research in Autism Spectrum Disorders, 7, 35 42. doi:10.1016/j.rasd.2012.05.002.

Mannion, A., \& Leader, G. (2013). An analysis of the predictors of comorbid psychopathology, gastrointestinal symptoms and epilepsy in children and adolescents with autism spectrum disorder. Research in Autism Spectrum Disorders, 7 (12), 1663-1671. doi:10.1016/j. rasd.2013.10.002.

Mannion, A., \& Leader, G. (in press). Gastrointestinal symptoms in Autism Spectrum Disorder: A literature review. Review Journal of Autism and Developmental Disorders. doi:10.1007/s40489-0130007-0.

Matson, J. L., \& Goldin, R. L. (2013). Comorbidity and autism: trends, topics, and future directions. Research in Autism Spectrum Disorders, 7, 1228-1233. doi:10.1016/j.rasd.2013.07.003.

Matson, J. L., \& González, M. L. (2007). Autism Spectrum DisordersComorbidity-Child Version. Baton Rouge, LA: Disability Consultants, LLC.

Matson, J. L., Rieske, R. D., \& Williams, L. W. (2013). The relationship between autism spectrum disorders and attention-deficit/hyperactivity disorder: an overview. Research in Developmental Disabilities, 34, 2475-2484. doi:10.1016/j.ridd.2013.05.021.

Mayes, S. D., \& Calhoun, S. L. (2009). Variable related to sleep problems in children with autism. Research in Autism Spectrum Disorders, 3, 931-941. doi:10.1016/j.rasd.2009.04.002.
Meltzer, L. J. (2011). Factors associated with depressive symptoms in parents of children with autism spectrum disorders. Research in Autism Spectrum Disorders, 5, 361-367. doi:10.1016/j.rasd.2010.05.001.

Meltzer, L. J., Montgomery-Downs, H. E., Insana, S. P., \& Walsh, C. M. (2012). Use of actigraphy for assessment in pediatric sleep research. Sleep Medicine Reviews, 16, 463-475. doi:10.1016/ j.smrv.2011.10.002.

Moon, E. C., Corkum, P., \& Smith, I. M. (2011). Case study: a case-series evaluation of a behavioral sleep intervention for three children with autism and primary insomnia. Journal of Pediatric Psychology, 36(1), 47-54. doi:10.1093/jpepsy/jsq057.

Owens, J. A., Nobile, C., McGuinn, M., \& Spirito, A. (2000). The Children's Sleep Habits Questionnaire: construction and validation of a sleep survey for school-aged children. Sleep, 23(8), 1043-51.

Park, S., Cho, S., Cho, I., Kim, B., Kim, J., Shin, M., et al. (2012). Sleep problems and their correlates and comorbid psychopathology of children with autism spectrum disorders. Research in Autism Spectrum Disorders, 6, 1068-1072. doi:10.1016/j.rasd.2012.02.004.

Richdale, A., Gavidia-Payne, S., Francis, A., \& Cotton, S. (2000). Stress, behaviour, and sleep problems in children with an intellectual disability. Journal of Intellectual \& Developmental Disability, 25(2), 147-161.

Rzepecka, H., McKenzie, K., McClure, I., \& Murphy, S. (2011). Sleep, anxiety and challenging behaviour in children with intellectual disability and/or autism spectrum disorder. Research in Developmental Disabilities, 2758-66. doi:10.1016/j.ridd.2011.05.034

Schreck, K. A. (1998). Preliminary analysis of sleep disorders in children with developmental disorders. Doctoral dissertation, The Ohio State University, 1997. Dissertation Abstracts International, 58, 3934.

Schreck, K. A., Mulick, J. A., \& Smith, A. F. (2004). Sleep problems as possible predictors of intensified symptoms of autism. Research in Developmental Disabilities, 25, 57-66. doi:10.1016/j.ridd. 2003.04.007.

Schreck, K. A., Mulick, J. A., \& Rojahn, J. (2003). Development of the Behavioral Evaluation of Disorders of Sleep scale. Journal of Child and Family Studies, 12(3), 349-359.

Sikora, D. M., Johnson, K., Clemons, T., \& Katz, T. (2012). The relationship between sleep problems and daytime behavior in children of different ages with autism spectrum disorders. Pediatrics, 130 (Supple. 2), S83-S90. doi:10.1542/peds.2012-0900F.

Sitnick, S. L., Goodlin-Jones, B. L., \& Anders, T. F. (2008). The use of actigraphy to study sleep disorders in preschoolers: some concerns about detection of nighttime awakenings. Sleep, 31 (3), 395-401.

Souders, M. C., Mason, T. B. A., Valladares, O., Bucan, M., Levy, S. E., Mandell, D. S., et al. (2009). Sleep behaviors and sleep quality in children with autism spectrum disorders. Sleep, 32(12), 1566-1578.

Spruyt, K., \& Gozal, D. (2011a). Pediatric sleep questionnaires as diagnostic or epidemiological tools: a review of currently available instruments. Sleep Medicine Reviews, 15, 19-32. doi:10.1016/j. smrv.2010.07.005.

Spruyt, K., \& Gozal, D. (2011b). Development of pediatric sleep questionnaires as diagnostic or epidemiological tools: a brief review of Dos and Don'ts. Sleep Medicine Reviews, 15, 7-17. doi:10.1016/j. smrv.2010.06.003.

Sparrow, S. S., Balla, D. A., \& Cicchetti, D. V. (1984). Vineland adaptive behavior scales: survey form manual. Circle Pines, MN: American Guidance Service.

Taylor, M. A., Schreck, K. A., \& Mulick, J. A. (2012). Sleep disruption as a correlate to cognitive and adaptive behavior problems in autism spectrum disorders. Research in Developmental Disabilities, 33, 1408-1417. doi:10.1016/j.ridd.2012.03.013.

Tsai, F., Chiang, H., Lee, C., Gau, S. S., Lee, W., Fan, P., et al. (2012). Sleep problems in children with autism, attention-deficit hyperactivity disorder, and epilepsy. Research in Autism Spectrum Disorders, 6, 413-421. doi:10.1016/j.rasd.2011.07.002.

Turner, K.S., \& Johnson, C.R. (2012). Behavioral interventions to address sleep disturbances in children with autism spectrum disorders: 
A review. Topics in Early Childhood Special Education, 1-9. doi:10.1177/0271121412446204

van de Wouw, E., Evenhuis, H. M., \& Echteld, M. A. (2012). Prevalence, associated factors, and treatment of sleep problems in adults with intellectual disability: a systematic review. Research in Developmental Disabilities, 33, 1310-1332. doi:10.1016/j.ridd. 2012.03.003.

van Golde, E. G. A., Gutter, T., \& de Weerd, A. W. (2011). Sleep disturbances in people with epilepsy; prevalence, impact, and treatment. Sleep Medicine Reviews, 15, 357-368. doi:10.1016/j.smrv. 2011.01.002.

Vgontas, A. N., \& Fernandez-Mendoza, J. (2011). Is there a link between mild sleep disordered breathing and psychiatric and psychosomatic disorders? Sleep Medicine Reviews, 15, 403-405. doi:10.1016/ j.smrv.2011.03.003.

Vriend, J. L., Corkum, P. V., Moon, E. C., \& Smith, I. M. (2011). Behavioral interventions for sleep problems in children with autism spectrum disorders: current findings and future directions. Journal of Pediatric Psychology, 36(9), 1017-1029. doi:10.1093/jpepsy/jsr044.

Weiskop, S., Richdale, A., \& Matthews, J. (2005). Behavioural treatment to reduce sleep problems in children with autism or fragile $\mathrm{X}$ syndrome. Developmental Medicine \& Child Neurology, 2, 94-104. doi:10.1017/S0012162205000186.

Wiggs, L., \& Stores, G. (2004). Sleep patterns and sleep disorders in children with autistic spectrum disorders: insights using parent report and actigraphy. Developmental Medicine \& Child Neurology, 46, 372-380. doi:10.1017/S0012162204000611.

Williams, K. C., Christofi, F. L., Clemmons, T., Rosenberg, D., \& Fuchs, G. J. (2012). Association of chronic gastrointestinal symptoms with sleep problems may help identify distinct subgroups of autism spectrum disorders. Gastroenterology, 5 (Suppl.1)(5), S-714. doi:10.1016/S0016-5085(12)62767-X.

Williams, G., Sears, L., \& Allard, A. (2006). Parent perceptions of efficacy for strategies used to facilitate sleep in children with autism. Journal of Developmental and Physical Disabilities, 18(1), 25-33. doi:10.1007/s10882-006-9003-y.

Wright, B., Sims, D., Smart, S., Alwazeer, A., Alderson-Day, B., Allgar, V., et al. (2011). Melatonin versus placebo in children with autism spectrum conditions and severe sleep problems not amenable to behaviour management strategies: a randomized controlled crossover trial. Journal of Autism and Developmental Disorders, 41, 175-184. doi:10.1007/s10803-010-1036-5. 